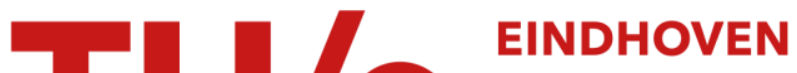 \\ UNIVERSITY OF \\ TECHNOLOGY
}

\section{Geometry of tracer trajectories in rotating turbulent flows}

\section{Citation for published version (APA):}

Alards, K. M. J., Rajaei, H., Del Castello, L., Kunnen, R. P. J., Toschi, F., \& Clercx, H. J. H. (2017). Geometry of tracer trajectories in rotating turbulent flows. Physical Review Fluids, 2(4), [044601].

https://doi.org/10.1103/PhysRevFluids.2.044601

DOI:

10.1103/PhysRevFluids.2.044601

Document status and date:

Published: 01/04/2017

\section{Document Version:}

Accepted manuscript including changes made at the peer-review stage

\section{Please check the document version of this publication:}

- A submitted manuscript is the version of the article upon submission and before peer-review. There can be important differences between the submitted version and the official published version of record. People interested in the research are advised to contact the author for the final version of the publication, or visit the $\mathrm{DOI}$ to the publisher's website.

- The final author version and the galley proof are versions of the publication after peer review.

- The final published version features the final layout of the paper including the volume, issue and page numbers.

Link to publication

\section{General rights}

Copyright and moral rights for the publications made accessible in the public portal are retained by the authors and/or other copyright owners and it is a condition of accessing publications that users recognise and abide by the legal requirements associated with these rights.

- Users may download and print one copy of any publication from the public portal for the purpose of private study or research.

- You may not further distribute the material or use it for any profit-making activity or commercial gain

- You may freely distribute the URL identifying the publication in the public portal.

If the publication is distributed under the terms of Article 25fa of the Dutch Copyright Act, indicated by the "Taverne" license above, please follow below link for the End User Agreement:

www.tue.nl/taverne

Take down policy

If you believe that this document breaches copyright please contact us at:

openaccess@tue.nl

providing details and we will investigate your claim. 


\title{
Geometry of tracer trajectories in rotating turbulent flows
}

\author{
Kim M. J. Alards,,${ }^{1,}$ Hadi Rajaei, ${ }^{1}$ Lorenzo Del Castello, ${ }^{2}$ Rudie \\ P. J. Kunnen, ${ }^{1}$ Federico Toschi, ${ }^{1,3,4}$ and Herman J. H. Clercx ${ }^{1}$ \\ ${ }^{1}$ Fluid Dynamics Laboratory, Department of Applied Physics, \\ Eindhoven University of Technology, P.O. Box 513, 5600 MB Eindhoven, The Netherlands \\ ${ }^{2}$ Istituto Sistemi Complessi, Consiglio Nazionale delle Ricerche, UOS Sapienza, 00185 Rome, Italy \\ ${ }^{3}$ Centre of Analysis, Scientific Computing, and Applications WEI, \\ Department of Mathematics and Computer Science, Eindhoven University of Technology, \\ P.O. Box 513, 5600 MB Eindhoven, The Netherlands \\ ${ }^{4}$ Istituto per le Applicazioni del Calcolo, Consiglio Nazionale delle Ricerche, Via dei Taurini 19, 00185 Rome, Italy
}

\begin{abstract}
The geometry of passive tracer trajectories is studied in two different types of rotating turbulent flows; rotating Rayleigh-Bénard convection (RBC; experiments and direct numerical simulations) and rotating electromagnetically forced turbulence (EFT; experiments). This geometry is fully described by the curvature and torsion of trajectories and from these geometrical quantities we can subtract information on the typical flow structures at different rotation rates. Previous studies, focusing on non-rotating homogeneous isotropic turbulence (HIT), show that the probability density functions (PDFs) of curvature and torsion reveal pronounced power laws. However, the set-ups studied here involve inhomogeneous turbulence and in RBC the flow near the horizontal plates is definitely anisotropic. We want to investigate how the typical shapes of the curvature and torsion PDFs, including the pronounced scaling laws, are influenced by this level of anisotropy and inhomogeneity and how this effect changes with rotation. A first effect of rotation is observed as a shift of the curvature and torsion PDFs towards higher values in the case of RBC and towards lower values in the case of EFT. This shift is related to the length scale of typical vortical structures that decreases with rotation in RBC, but increases with rotation in EFT, explaining the opposite shifts of the curvature (and torsion) PDFs. A second remarkable observation is that in RBC the HIT scaling laws are always recovered, as long as the boundary layer (BL) is excluded. This suggests that these scaling laws are very robust and hold as long as we measure in the turbulent bulk. In the BL of the RBC cell, however, the scaling deviates from the HIT prediction for lower rotation rates. This scaling behavior is found to be consistent with the coupling between the boundary layer dynamics and the bulk flow, that changes under rotation. In particular, it is found that the active coupling of the Ekman-type boundary layer with the bulk flow suppresses anisotropy in the BL region for increasing rotation rates.
\end{abstract}

\section{INTRODUCTION}

Rotating turbulence is important not only in astro- and geophysical flows [1, 2, but also in many industrial applications that involve flow in rotating components [3, 4. An important effect of rotation is the development of columnar structures in the flow, with characteristic time- and length-scales depending on the rotation rate 5 -7. Depending on the type of turbulence, rotation can have additional effects, such as a transition to enhanced heat flux in rotating turbulent convection [8 12]. We therefore investigate two different turbulent flow configurations in this research: rotating (isothermal) turbulence driven by electromagnetic forcing and rotating turbulent Rayleigh-Bénard convection (RBC). In RBC the flow is buoyancy driven: a fluid is heated from below and cooled from above. Rotating a RBC system around its vertical axis induces sharp transitions in the heat flux and boundary layer dynamics and a significant change of the dominant coherent flow structures [8, 13, 15.

Studying the effect of rotation on coherent flow structures via a Lagrangian method, i.e. by acquiring statistical data sets by tracking individual fluid particles, is shown to be an interesting approach [16, 17]. Lagrangian measurements are often in the form of velocity and acceleration time series, however also the shape of particle trajectories themselves provides essential information on the geometrical aspects of the flow [18 20. When a trajectory is considered as a curve in 3D space, its geometry is fully described by its curvature and torsion.

So far, such curvature and torsion measurements have focused only on homogeneous isotropic turbulence (HIT) [1821 and these measurements have not been used to study particle trajectories in rotating turbulence. In HIT, curvature and torsion PDFs reveal pronounced power laws, which was shown both experimentally [20] and numerically [19, 21]. These power laws can be derived analytically, assuming that the velocity components in turbulence are Gaussian

\footnotetext{
* k.m.j.alards@tue.nl
} 
random variables 20. This is however not the case in the turbulent flows studied here, since the PDFs of vertical velocity are shown to have wider tails, compared to the Gaussian distribution, in both electromagnetically forced turbulence (EFT) and Rayleigh-Bénard convection (RBC) [22, 23. Moreover, anisotropy and inhomogeneity play a role, especially in the flow close to the horizontal top and bottom plates [22 25]. Even though the scaling laws, derived in HIT, are shown to be robust 20, it is not clear how anisotropy, inhomogeneity and non-Gaussian velocity statistics influence the curvature and torsion PDFs and whether one recovers these scaling laws in different turbulent flows like, for example, the ones studied here.

Also the effect of rotation on curvature and torsion statistics is not obvious. First, rotation does influence the level of anisotropy and inhomogeneity in RBC and EFT [22 25]. Second, it changes the length scales of the typical coherent structures in both types of turbulence systems. This is expected to affect the statistics of the trajectories of tracer particles.

So far we disregarded the boundary layer and discussed effects related to the turbulent flow in the bulk of the RBC and EFT systems. The boundary layer is a special region of the flow, where the typical statistical characteristics of bulk turbulence are not applicable anymore [26, 27. In RBC, the boundary layer dynamics is significantly influenced by rotation [13, 14 and moreover plays an important role in the transition to the enhanced heat flux state, mentioned before [8, 15. More precisely, a transition is observed from a Prandtl-Blasius boundary layer for lower rotation rates, to an Ekman boundary layer for higher rotation rates [13. A significant difference between these two types of boundary layers is that the Prandtl-Blasius boundary layer is passive and does not interact with the bulk, while the Ekman boundary layer dynamics is actively driven by the bulk flow. Therefore we expect the behavior of curvature and torsion statistics in the Ekman boundary layer to approach the bulk behavior at higher rotation rates. This can be studied by comparing scaling laws, measured in the bulk, to scaling laws measured in the boundary layer at different rotation rates. In the bulk we will moreover consider a measurement volume in the center of the cell and one closer to the top plate, to include effects of anisotropy and inhomogeneity 22 25]. In this way, we aim at finding a general effect of rotation on the geometry of particle trajectories, not only in different types of turbulent flows, but also closer to, or even inside, the boundary layer.

In this paper we will first give a theoretical background on curvature and torsion and show how the probability density functions (PDFs) are expected to scale in the limit of high and low curvature and torsion values. Then, in section III, we will briefly discuss experimental and numerical methods. We will show results in the form of curvature and torsion PDFs, collected at different rotation rates, in section IV. Here we will moreover include statistics measured in the boundary layer at the cold top plate in RBC. In section $\mathrm{V}$ we will summarize and conclude our findings.

\section{SCALING BEHAVIOR OF THE CURVATURE AND TORSION PDFS}

When a tracer trajectory is considered as a curve in 3D space, its geometry is described by two fundamental geometrical parameters, the curvature, $\kappa$, and the torsion, $\tau$. These parameters depend on the velocity, $\mathbf{u}$, and acceleration, a, of the tracer as:

$$
\begin{aligned}
\kappa & =\frac{|\mathbf{u} \times \mathbf{a}|}{|\mathbf{u}|^{3}}, \\
\tau & =\frac{\mathbf{u} \cdot(\mathbf{a} \times \dot{\mathbf{a}})}{(\mathbf{u} \cdot \mathbf{u})^{3} \kappa^{2}} .
\end{aligned}
$$

In previous studies curvature statistics were collected in homogeneous isotropic turbulence (HIT) both experimentally and numerically [18 20. The tails of the curvature PDFs were shown to reveal pronounced power laws, scaling as $\kappa^{1}$ for low curvature values and as $\kappa^{-5 / 2}$ for high curvature values [18, 20. In order to explain these power laws analytically it is convenient to use the alternative formulation $\kappa=a_{n} u^{-2}$, with $a_{n}$ the absolute value of the component of the acceleration normal to the velocity [20]. In an isotropic turbulent flow the velocity components are assumed to be independent random Gaussian variables and as a result the probability distribution of $u^{-2}$ follows an inverse $\chi$-squared distribution of dimension $k=3$. The normal acceleration has only two components and therefore follows a $\chi$-distribution of dimension $k=2$, under the assumption that velocity and acceleration are uncorrelated. As a result the distribution of $u^{-2}$ and $a_{n}$ obey:

$$
\begin{aligned}
P_{u^{-2}}(x ; k) & =\frac{2^{-\frac{k}{2}} x^{-\frac{k}{2}-1} \mathrm{e}^{-\frac{1}{2 x}}}{\Gamma\left(\frac{k}{2}\right)}, \\
P_{a_{n}}(z ; k) & =\frac{2^{1-\frac{k}{2}} z^{k-1} \mathrm{e}^{-\frac{z^{2}}{2}}}{\Gamma\left(\frac{k}{2}\right)},
\end{aligned}
$$


where $\Gamma(k / 2)$ denotes the Gamma function $[28$.

The statistics of $u \kappa$ and $\kappa$, shown in [18, and the correlation between $\kappa$ and $u$ (and $a$ ), shown in 20, 21, reveal that large curvature values correspond to small $u^{2}$ (i.e. large $u^{-2}$ ) at finite $a_{n}$. We expand the distribution $P_{u^{-2}}\left(x=u^{-2}\right)$ to $x$ for $x \rightarrow \infty$, resulting in $P_{u^{-2}}\left(x=u^{-2}\right) \sim x^{-5 / 2}$. So, in the limit of $\kappa \rightarrow \infty$ the curvature PDF is expected to scale as $P_{\kappa}(\kappa) \sim \kappa^{-5 / 2}$. On the opposite, low curvature values correspond to small normal acceleration at finite velocity. When expanding $P_{a_{n}}\left(z=a_{n}\right)$ (see equation (4p) in $z$, for $z \rightarrow 0$, one obtains $P_{a_{n}}\left(z=a_{n}\right) \sim z$. This means that in the limit of $\kappa \rightarrow 0$ the curvature PDF is expected to scale as $P_{\kappa}(\kappa) \sim \kappa$.

Also for the torsion PDFs pronounced power laws were found, corresponding to a $\tau^{-3}$ scaling in the high torsion limit [19, 21. Again Gaussian analysis can be used to derive this scaling, now based on $\tau=|\dot{\mathbf{a}} \cdot \hat{\mathbf{n}}| /\left(a_{n} u\right)$, with $\hat{\mathbf{n}}$ the unit vector normal to the velocity. When assuming that $a_{n}$ and $u$ are uncorrelated, that $|\dot{\mathbf{a}} \cdot \hat{\mathbf{n}}|$ is finite and that $a_{n}$ and $u$ follow a $\chi$-distribution of respectively dimensions 2 and 3, the PDF of the torsion becomes [19]:

$$
P_{\tau}(\tau) \sim \iint u^{2} \mathrm{e}^{-\frac{u^{2}}{2}} a_{n} \mathrm{e}^{-\frac{a_{n}^{2}}{2}} \delta\left(\tau-\frac{1}{a_{n} u}\right) d a_{n} d u,
$$

where $\delta()$ is Dirac's delta function. We first solve this integral for $u$, using the properties of the delta function, and introduce a term $\beta=1 / \tau^{2}$ :

$$
P_{\tau}(\tau) \sim-\beta^{2} \int_{0}^{\infty} \frac{1}{a_{n}^{2}} \mathrm{e}^{-\frac{\beta}{2 a_{n}^{2}}} \mathrm{e}^{-\frac{a_{n}^{2}}{2}} d a_{n}=-\beta^{2} G
$$

Upon defining $F=\int_{0}^{\infty} \mathrm{e}^{-a_{n}^{2} / 2} \mathrm{e}^{-\beta /\left(2 a_{n}^{2}\right)} d a_{n}$ we obtain $G=-2 d F / d \beta$. One can also rewrite the integral $G$ in terms of a new variable $y=\sqrt{\beta} / a_{n}$, using $d a_{n}=-d y \sqrt{\beta} / y^{2}$ :

$$
G=\int_{0}^{\infty} \frac{1}{\sqrt{\beta}} \mathrm{e}^{-\frac{\beta}{2 y^{2}}} \mathrm{e}^{\frac{-y^{2}}{2}} d y
$$

Note that the right-hand side of this equation equals $F / \sqrt{\beta}$ which gives, combined with $(6)$, the following differential equation: $d F / d \beta=-F /(2 \sqrt{\beta})$. The solution is of the form $F=F_{0} \mathrm{e}^{-\sqrt{\beta}}$, with $F_{0}$ being a constant. This can be substituted into equation (6) resulting in:

$$
P_{\tau}(\tau) \sim 2 \beta^{2} \frac{d F}{d \beta} \sim-\beta^{3 / 2} F_{0} \mathrm{e}^{-\sqrt{\beta}} .
$$

Using the definition $\beta=1 / \tau^{2}$, we find that in the limit of $\tau \rightarrow \infty$ the torsion $\operatorname{PDF}, P_{\tau}(\tau)$, scales as $P_{\tau}(\tau) \sim \tau^{-3}$. In the limit of $\tau \rightarrow 0$ the torsion PDF scales as $\tau^{0}$ [18, 19, however a theoretical explanation for this scaling is lacking.

\section{EXPERIMENTAL AND NUMERICAL METHODS}

\section{A. Rayleigh-Bénard convection}

First, we introduce the Rayleigh-Bénard setup, that is described in detail in [13, 23. The setup includes a convection cell and an optical tracking system, both mounted on a rotating table. The convection cell consists of a cylindrical Plexiglas vessel, filled with water, with height and diameter equal to $H=D=200 \mathrm{~mm}$, resulting in an aspect ratio of $\Gamma=D / H=1$. At the bottom a copper plate with an electrical resistance heater is attached and at the top a transparent cooling chamber is placed. Neutrally buoyant polyethylene particles, with a diameter of $d_{p}=75-90 \mu \mathrm{m}$ and a density of $\rho_{p}=1002 \mathrm{~kg} \mathrm{~m}^{-3}$, are inserted in the working fluid that has a density of $\rho_{f}=998 \mathrm{~kg} \mathrm{~m}^{-3}$. The resulting Stokes number of the particles, defined as $S t=\tau_{p} / \tau_{\eta}$, with $\tau_{p}=d_{p}^{2} \rho_{p} /\left(18 \nu \rho_{f}\right)$ (where $\nu$ is the kinematic viscosity) and $\tau_{\eta}$ the Kolmogorov time scale, is $S t \approx 3 \cdot 10^{-4}$, which means that the particles will behave as tracers. We however realize that the particles are on average heavier than the fluid, resulting in a positive settling velocity of $w=\left(\rho_{p}-\rho_{f}\right) g d_{p}^{2} /\left(18 \nu \rho_{f}\right) \approx 0.0183 \mathrm{~ms}^{-1}$, where $g$ is the gravitational acceleration. The system is illuminated by four arrays of LEDs. Particle images are recorded by four CCD cameras with a resolution of $1600 \times 1200$ pixels, at a frequency of $30 \mathrm{~Hz}$. Trajectories, reconstructed from the images using the 3D-Particle Tracking Velocimetry (3D-PTV) algorithm developed at ETH Zürich [29, 30, are filtered using 3D cubic polynomial fitting [31. The dimensionless numbers characterizing the (turbulent) flow in the rotating RBC setup are the Rayleigh number, $R a=\alpha g \Delta T H^{3} /(\sigma \nu)$, the Prandtl number, $\operatorname{Pr}=\nu / \sigma$ and the Ekman number $E k=\nu /\left(2 \Omega H^{2}\right)$, where $\sigma$ is the thermal diffusivity of the 
fluid, $\Delta T$ the applied temperature difference between top and bottom plate, $\Omega$ the rotation rate and $\alpha$ the thermal expansion coefficient. In this work $R a=1.28 \cdot 10^{9}$, resulting from an applied temperature difference of $\Delta T=10 \mathrm{~K}$, $\operatorname{Pr}=6.7$ and the rotation rate is varied from $E k=\infty\left(0 \mathrm{rad} \mathrm{s}^{-1}\right)$ to $E k=7.2 \cdot 10^{-6}\left(1.65 \mathrm{rad} \mathrm{s}^{-1}\right)$. Moreover, two different measurement volumes are used as sketched in figure 1. one in the center of the cell (gray cuboid), referred to as center measurement volume, and one close to the top plate (hatched cuboid), referred to as top measurement volume, both of size $80 \times 60 \times 50 \mathrm{~mm}^{3}$ (in Cartesian coordinates) and centered around the cylinder axis. In the experiments we cannot treat the boundary layer (BL) separately, however we realize that the number of measurement points inside the BL is minimal due to particles being slightly heavier than the fluid and due to an offset of about $1 \mathrm{~mm}$ between the plate and the field of view of the cameras. In terms of the viscous boundary layer thickness $\delta_{u}$, that is determined as the position of the maximum horizontal rms velocity as in [13], this corresponds to $0.167 \delta_{u}$ for $E k=\infty$ and $0.617 \delta_{u}$ for $E k=7.2 \cdot 10^{-6}$. As a result, we can assume that the statistics measured experimentally in the top measurement volume, behave as if we are excluding the BL (as in the light-green volume in figure 1 .

As explained in [20] high curvature events correspond to particles that suddenly change their direction of motion. These sudden motions of particles are difficult to capture in post-processing of subsequent images of particle tracking. Also due to the filtering of trajectories extreme acceleration and extreme curvature events are not always measured accurately in the experiments and it is not clear how this might affect our results. Torsion is even more difficult to measure experimentally, as it includes the time derivative of acceleration. In order to also include extreme curvature events, torsion statistics and to have access to the boundary layer, we complement the RBC experiments with direct numerical simulations (DNS), modeling the exact same cylindrical system. The governing dimensionless equations are the incompressible Navier-Stokes and energy equations in the Boussinesq approximation:

$$
\begin{aligned}
\nabla \cdot \mathbf{u} & =0 \\
\frac{\partial \mathbf{u}}{\partial t}+(\mathbf{u} \cdot \nabla) \mathbf{u}+\frac{1}{E k} \sqrt{\frac{P r}{R a}} \hat{\mathbf{z}} \times \mathbf{u} & =-\nabla p+\sqrt{\frac{P r}{R a}} \nabla^{2} \mathbf{u}+T \hat{\mathbf{z}}, \\
\frac{\partial T}{\partial t}+(\mathbf{u} \cdot \nabla) T & =\frac{1}{\sqrt{P r R a}} \nabla^{2} T
\end{aligned}
$$

with $\mathbf{u}$ the velocity vector, $t$ time, $p$ pressure, $T$ temperature and $\hat{\mathbf{z}}$ the vertical unit vector. These equations are non-dimensionalized using the cell height $H$ for length, $\Delta T$ for temperature and $t_{c}=H / U$ for time, based on the freefall velocity $U \equiv \sqrt{g \alpha \Delta T H}$. These equations are solved in cylindrical coordinates with no-slip boundary conditions (BCs) at all walls, a fixed temperature BC at the top/bottom horizontal walls and adiabatic BCs (i.e. no heat flux) at the sidewalls. We use a resolution of $512 \times 384 \times 512$ grid points in the azimuthal, radial and axial direction, respectively. To ensure at least ten grid points within the boundary layer, grid refinement is used in both the vertical and radial directions. To discretize this set of equations a second-order finite difference scheme is applied and for the integration a third-order Runge-Kutta method is chosen. Details of the numerical scheme can be found in [32, 33. Just as in the experiments, tracer particles, following the fluid motion exactly, are tracked. To interpolate the fluid velocity from the surrounding eight grid points around the particle position, we use a tri-linear interpolation scheme and for the time integration a second-order Adams-Bashforth scheme is used. In total $10^{6}$ particles are evolved in the system. To allow direct comparison with experiments we collect statistics in the same measurement volumes as in the experiments. However, in the DNS the top measurement volume is subdivided into the boundary layer, referred to as BL region, and a region excluding the BL, referred to as non-BL region, as sketched in figure 1 , As mentioned before the boundary layer thickness is determined as the position of the maximum horizontal rms velocity, as in [13]. Furthermore, the control parameters are set as in the experiments, only the Ekman range is extended with $E k=7.2 \cdot 10^{-4}$ and $E k=3.6 \cdot 10^{-6}$, such that Ekman varies between $E k=\infty$ and $E k=3.6 \cdot 10^{-6}$.

\section{B. Electromagnetically driven turbulence}

Next to the RBC experiments we also study rotating (isothermal) turbulence driven by electromagnetic forcing. Our setup is described in [22, 34. Briefly, it consists of a transparent tank, a turbulence generator and a 3D-PTV system. The Perspex cubic tank, with inner dimensions of $500 \times 500 \times 250 \mathrm{~mm}^{3}$, is filled with an electrolyte solution with density $\rho_{f}=1.19 \cdot 10^{3} \mathrm{~kg} \mathrm{~m}^{-3}$ and kinematic viscosity $\nu=1.319 \mathrm{~mm}^{2} \mathrm{~s}^{-1}$. The flow is driven by an electromagnetic forcing system, consisting of a magnet array arranged according to a chessboard pattern as discussed in [22, 34]. The maximum forcing length is determined by the spacing between the magnets, which equals $L=70 \mathrm{~mm}$. Poly methyl methacrylate (PMMA) particles with a diameter of $d_{p}=127 \pm 3 \mu \mathrm{m}$ and a density of $\rho_{p}=1.19 \cdot 10^{3} \mathrm{~kg} \mathrm{~m}^{-3}$ are inserted in the fluid. The Stokes number of these particles is $S t \sim 10^{-3}$ and the settling velocity approaches zero due to the match of fluid and particle density. This means that also in the EFT experiments particles behave as tracers. 


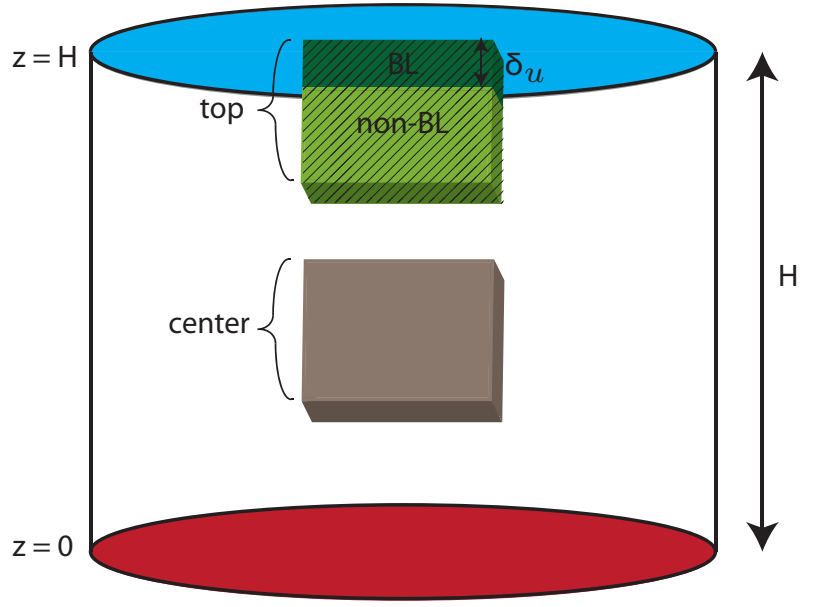

Figure 1: Sketch of the measurement volumes. The gray cuboid represents the center measurement volume of size $0.4 H \times 0.3 H \times 0.25 H$, with $H=200 \mathrm{~mm}$ being the cell height. The green hatched cuboid represents the top measurement volume, near the top plate, which is subdivided in the vertical direction into a non-boundary layer (non-BL) part of size $0.4 H \times 0.3 H \times\left(0.25 H-\delta_{u} H\right)$ (light-green), and a boundary layer (BL) part of size $0.4 H \times 0.3 H \times \delta_{u} H\left(\right.$ dark-green), with $\delta_{u}$ the dimensionless viscous boundary layer thickness, which varies between $\delta_{u}=0.0299$ and $\delta_{u}=0.0058$, depending on the rotation rate 13 . This subdivision in BL and non-BL is only applied in the DNS, while in the experiments the total top measurement volume is used, as represented by the total green hatched volume. Note that the size of the BL region is not real-scale here but rather enlarged for visibility reasons.

These neutrally buoyant particles are tracked by four high speed cameras with a resolution of $1024 \times 1024$ pixels and images are processed using the 3D-PTV and filtering procedure as described previously for RBC. The complete setup is mounted on a rotating table, of which the rotation axis coincides with the tank vertical axis. Depending on the rotation rate the time step for data acquisition is $\Delta t=16.7 \mathrm{~ms}$ (rotation rates below $2.0 \mathrm{rad} \mathrm{s}^{-1}$ ) or $\Delta t=33.3 \mathrm{~ms}$ (rotation rates greater than $2.0 \mathrm{rad} \mathrm{s}^{-1}$ ). The field of view is of size $100 \times 100 \times 100 \mathrm{~mm}^{3}$, centered in the horizontal plane and starting right above the bottom plate. Rotation is again characterized by the Ekman number, now based on the forcing length $L$ as $E k=\nu /\left(2 \Omega L^{2}\right)$ and is varied between $E k=\infty$ (no rotation) and $E k=2 \cdot 10^{-6}\left(5.0 \mathrm{rad} \mathrm{s}^{-1}\right)$. The Taylor-scale Reynolds number $\left(R e_{\lambda}=u_{r m s} \lambda / \nu\right.$, with $u_{r m s}$ the root-mean-square of the velocity fluctuations and $\lambda$ the Taylor micro-scale) is in the range of $70 \leq R e_{\lambda} \leq 110$ depending on the rotation rate.

\section{RESULTS}

\section{A. Non-rotating turbulence}

We first discuss curvature and torsion measurements in non-rotating turbulence and investigate whether we recover the scaling laws derived for HIT in section [II For RBC we start these measurements in the cell center, where the flow is closest to HIT [23, 25, 35]. However we know that, even in the center of the cell, the vertical velocity PDF shows non-Gaussian behavior 23. Also in EFT the flow is not perfectly homogeneous, but is shown to be inhomogeneous in the vertical direction and homogeneous in the horizontal direction 22]. The resulting curvature PDFs, from both experiments and DNS, are shown in figure 2a, where we normalize data with the cell height $H$, in the case of turbulent convection, and with the forcing length $L$, in the case of electromagnetic forcing. Both $H$ and $L$ are fixed during the experiments and simulations, and therefore this non-dimensionalization allows for comparison between the two types of turbulent flows and between different rotation rates. A good agreement between all three curvature PDFs is found within the error margins discussed in the caption. This means that not only DNS and experiments on RBC agree very well, but also that similar (dimensionless) curvature statistics are measured in the two completely different setups in the absence of rotation. Moreover, the HIT power laws, represented by the black lines, are recovered in both turbulence systems despite the vertical inhomogeneity in the EFT setup and non-Gaussian vertical velocity statistics in RBC. A possible explanation is that the scaling laws are derived in the limit of small velocity, where the assumption of Gaussian statistics is again reasonable. Indeed we checked from our data that the PDFs of vertical velocity are Gaussian around the peak at $u=0$ and show non-Gaussian behavior only in high velocity tails of the PDFs. We also computed the joint statistics between velocity and acceleration and observed that in the bulk velocity and acceleration are uncorrelated, confirming that the assumptions made in section $\Pi$ II are not heavily violated. For 


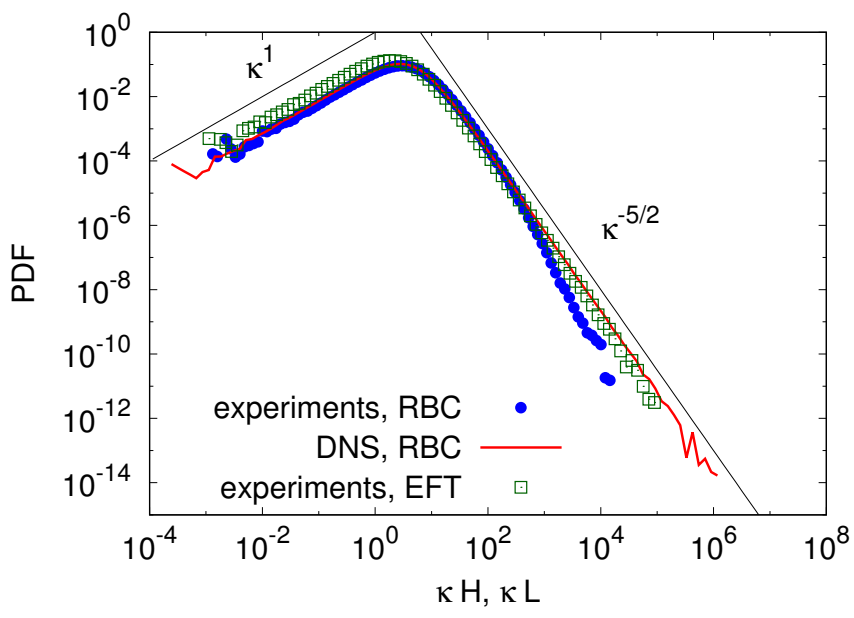

(a) curvature

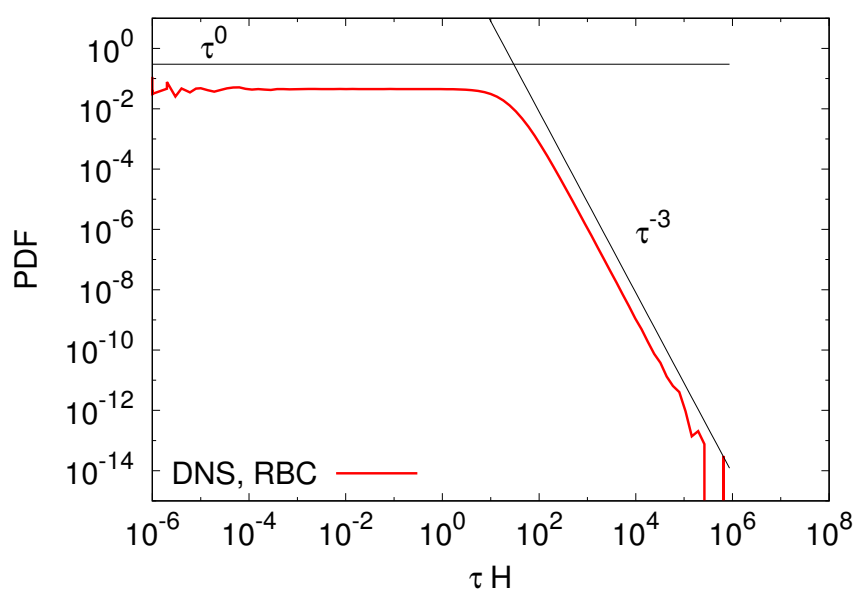

(b) torsion

Figure 2: (a) Curvature, $\kappa$, PDFs for both Rayleigh-Bénard convection (RBC, experiments and DNS in the cell center) and turbulence driven by electromagnetic forcing (EFT, experiments). Curvature is non-dimensionalized using the cell height, $H$, for RBC and the forcing length, $L$, for EFT. (b) Torsion, $\tau$, PDF, measured numerically in the center of the convection cell. Torsion is non-dimensionalized using the cell height, $H$. Errors of curvature and torsion PDFs, in the center of the RBC cell, are always estimated as the deviation between the PDF, constructed in the upper half of the measurement volume, and the PDF, constructed in the lower half of the measurement volumes (disregarding the outer parts of the tails, where the error is larger). Here, torsion and curvature PDFs measured with DNS have an estimated relative error of $4 \%$, while curvature PDFs measured experimentally have a larger relative error of about $20 \%$ for $\kappa H>10^{3}$ and $8 \%$ for $\kappa H<10^{3}$. PDFs measured in EFT, where we subdivide the measurement volume in a right and left sub-volume (rather than top- and bottom as in the bulk) to compute the error, have a relative error of $5 \%$

the $\mathrm{RBC}$ experiments the $\kappa^{-5 / 2}$ scaling is recovered up to $\kappa H \approx 10^{3}$, although the PDF deviates from this scaling for larger curvature values. The latter is the result of the experimental particle-tracking procedure in which sudden movements of particles, corresponding to extreme curvature events, results in loss of these particles. This effect is also reflected in the relative error of the curvature PDF in the RBC experiments, computed by subdividing the measurement volume vertically and comparing PDFs constructed in these two sub-volumes. For curvature values of $\kappa H>10^{3}$ the relative error is $20 \%$, while for curvature values of $\kappa H<10^{3}$ it is reduced to $8 \%$. In the EFT experiments extreme curvature events are captured more accurately due to different camera settings, illumination protocol and different particle properties [22, allowing for more reliable particle tracking between subsequent time steps. The numerical torsion PDF for non-rotating convection is shown in figure 2b, together with the $\tau^{-3}$ scaling law (black solid line). Also for torsion the predicted power law is recovered very well in the center of the convection cell. We moreover observe that for low torsion values the PDF scales as $\tau^{0}$, which is in agreement with the result in [19, 21].

We continue by analyzing curvature and torsion statistics in the top measurement volume of the RBC cell, where we expect the flow to be anisotropic. Near the top plate we distinguish between the (viscous) BL region and the non-BL layer region (figure 1). In the DNS a sufficient amount of tracer particles will penetrate the boundary layer, while in the experiments we expect very few particles to enter the boundary layer, since the particles are not perfectly neutrally buoyant. In figure 3a we show the curvature PDFs, measured near the top plate. Now we do not have vertical symmetry within the measurement volume and relative errors are computed by subdividing the total measurement volume horizontally into two equal parts and comparing PDFs constructed in these sub-volumes. We observe in figure $3 \mathrm{a}$ that the PDF obtained from the experiments perfectly matches the PDF from the numerical simulations, but measured outside the BL region. While these PDFs recover the $\kappa^{-5 / 2}$ scaling law, the PDF measured numerically inside the boundary layer shows a different scaling behavior. For the torsion PDFs, shown in figure 3b we again observe that the PDF constructed in the non-BL region recovers the HIT scaling law, while the PDF constructed in the BL region reveals a different scaling behavior. So, even though the bulk flow near the top plate is anisotropic [8, 25, this does not influence the scaling behavior of the curvature and torsion PDFs. This suggests that as long as viscosity does not play a significant role and as long as the flow is turbulent, the scaling laws found in section II hold. In the boundary layer, however, we clearly see an effect of the BL dynamics of the flow on the large-curvature scaling. At the end of the following paragraph, we discuss this point in more detail and show the effect of rotation on 


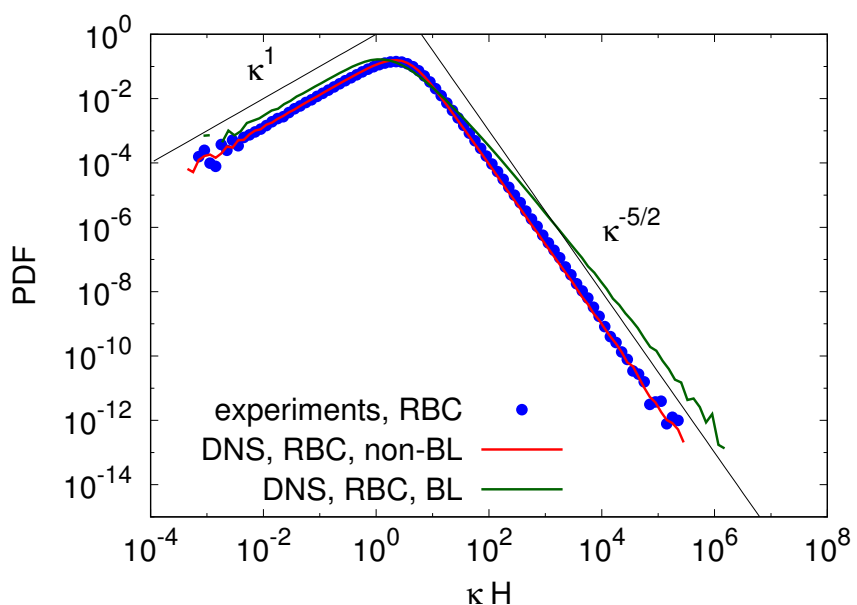

(a) curvature

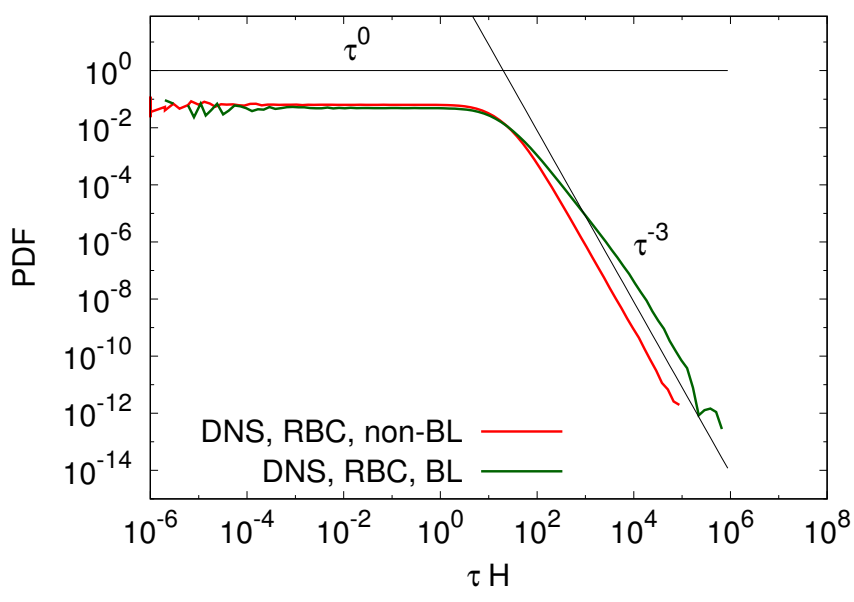

(b) torsion

Figure 3: (a) Curvature, $\kappa$, PDFs for Rayleigh-Bénard convection (RBC, experiments and DNS at the top of the cell). (b) Torsion, $\tau$, PDFs, measured numerically at the top of the convection cell. In the DNS the top measurement volume is subdivided into the non-boundary layer (non-BL) region and the boundary layer (BL) region (see figure 1). Curvature and torsion are non-dimensionalized using the cell height, $H$. Errors of curvature and torsion PDFs, near the top plate of the RBC cell, are always estimated by subdividing the measurement volumes horizontally into two equal parts and computing the deviation between the PDFs, constructed in these two sub-volumes (disregarding the outer parts of the tails, where the error is larger). Torsion and curvature PDFs outside the BL, measured with DNS, have an estimated relative error of $6 \%$, while the error of PDFs inside the BL can go up to $10 \%$ Curvature PDFs measured experimentally in the top measurement volume have a relative error of $5 \%$.

this scaling behavior in the boundary layer.

\section{B. Rotating turbulence}

We now consider the effect of rotation on the curvature and torsion statistics, which are measured and computed for a wide range of Ekman numbers. The PDFs, measured in electromagnetically forced turbulence, are shown in figure $4 \mathrm{a}$. Both the $\kappa^{1}$ and the $\kappa^{-5 / 2}$ scalings are recovered for all Ekman numbers. The effect of rotation is observed as a shift of the complete curve towards lower curvature values. For RBC, we first compute the PDFs in the center of the convection cell, which are shown in figure $4 \mathrm{~b}$ and $4 \mathrm{c}$ for experiments and DNS, respectively. In both figures, we observe a shift of the PDFs towards higher curvature values, opposite to the shift observed in EFT. The normalized curvature PDFs, shown in figure 4d, will be discussed below, where we address the observed shift in more detail. As in EFT, the scaling laws are unaffected by rotation in the DNS. In the RBC experiments, the $\kappa^{-5 / 2}$ scaling is not recovered for $\kappa H \gtrsim 10^{3}$, which is a result of the loss of high curvature events as explained in section III A.

Measurements near the top plate, where we expect a larger effect due to statistical anisotropy and where viscosity might come into play, are shown in figure 5. In the experiments and in the DNS which focus on the non-BL region, we observe a similar shift towards higher values of $\kappa$, as observed in the center. Moreover, we recover the $\kappa^{-5 / 2}$ scaling for all rotation rates. In the boundary layer, where we can measure only numerically, we clearly distinguish two regimes in figure $5 \mathrm{c}$ In the first regime, where $E k>1.8 \cdot 10^{-4}$, the scaling is less steep than $\kappa^{-5 / 2}$, while for $E k \lesssim 1.8 \cdot 10^{-4}$ the scaling is closer to $\kappa^{-5 / 2}$. We also observe a clear shift of the PDFs towards larger curvature under rotation.

As a reference point for the shift of the PDFs, we use the position of the maximum, corresponding to the most probable value of curvature. We compute this maximum position, indicated by $\kappa^{*}$, for all PDFs from figures 4 (a)-(c) and 5 (a)-(b) and plot the results as a function of the Ekman number in figure $5 \mathrm{~d}$. In the DNS, we focus on the non-BL region of the top measurement volume, where we can compare the DNS to the experimental results. Indeed, a perfect agreement between experiments and DNS is found for the peak position $\kappa^{*}$ in figure $5 \mathrm{~d}$, both in the center and near the top plate.

The shifts of the PDFs are expected to be related to the development of typical coherent flow structures, which are vortices in turbulent flows with a characteristic length scale. For electromagnetically driven turbulence the length scale of these typical vortical structures increases with rotation up to a rotation rate of $\Omega=1.0 \mathrm{rad} \mathrm{s}^{-1}\left(E k=1 \cdot 10^{-5}\right)$ 


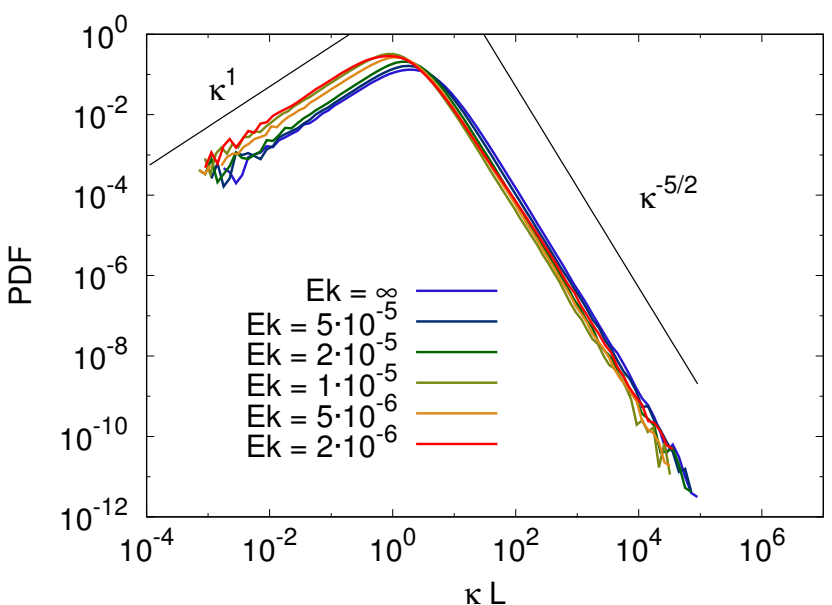

(a) experiments, EFT

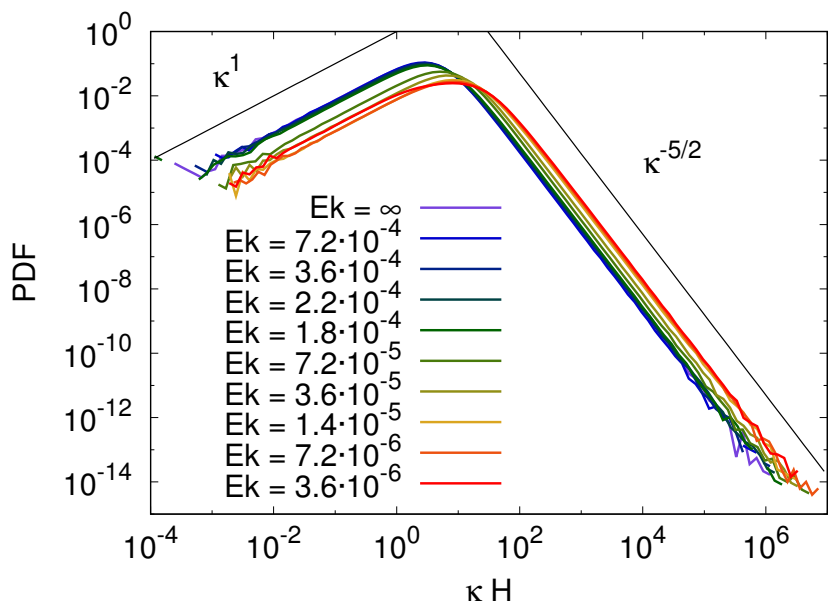

(c) numerics, $\mathrm{RBC}$

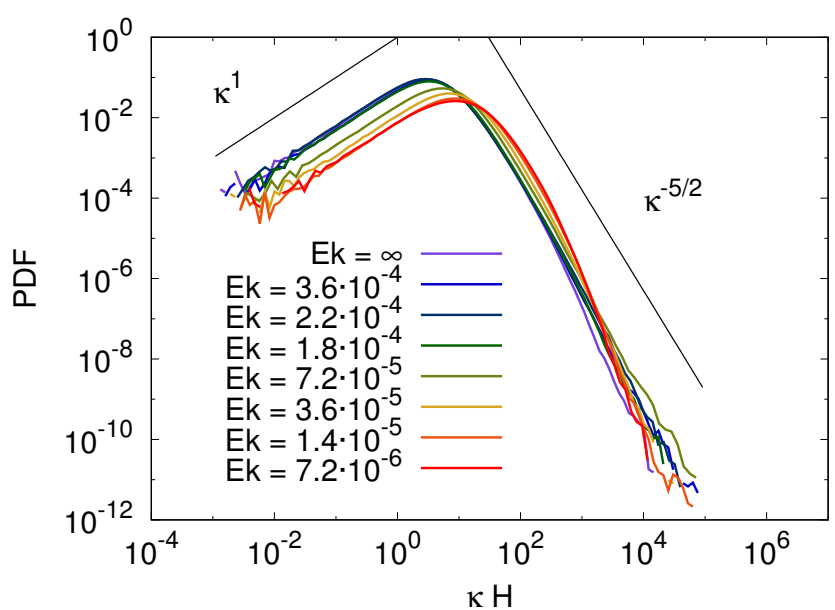

(b) experiments, RBC

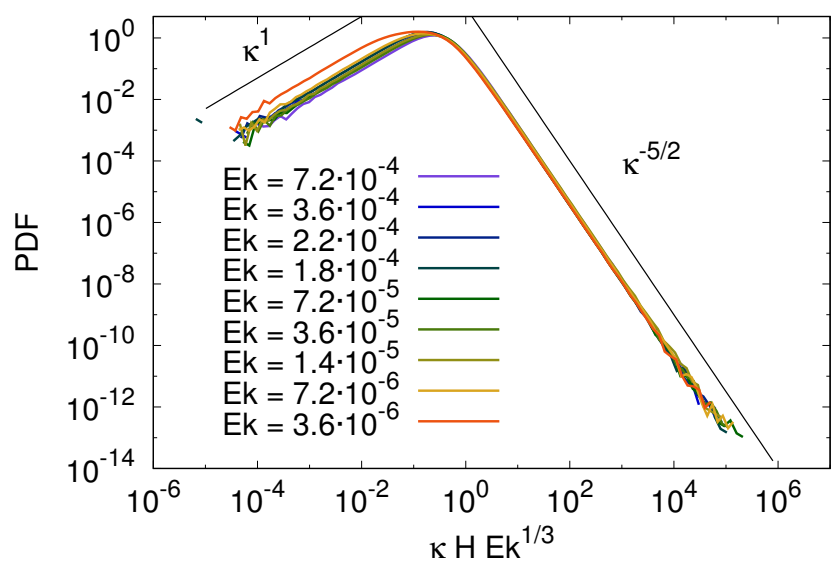

(d) numerics, RBC

Figure 4: (a) Curvature, $\kappa$, PDFs measured in electromagnetically forced turbulence (EFT), for different Ekman numbers. Curvature is non-dimensionalized using the forcing length, L. Focusing on the left part of the curve, the Ekman number is increasing from top to bottom. (b)-(c) Curvature PDFs, measured experimentally (b) and numerically (c) in Rayleigh-Bénard convection (RBC), in the center of the RBC cell, for different Ekman numbers. (d) PDFs of figure (c), but now normalized by $E k^{1 / 3}$. Because of this normalization the PDF for $E k=\infty$ is not shown. Focusing on the left part of the curves of panels (b)-(d), the Ekman number is increasing from bottom to top. For RBC, curvature is non-dimensionalized using the cell height, $H$. Errors in the PDFs are as discussed in figure 2 .

[5, 22, 36 and since curvature is an inverse length we expect an opposite trend for the peak position in this regime. This increase in length scale is limited by the forcing length, which corresponds to $\kappa^{*} L=1$. In figure $5 \mathrm{~d}$ we indeed observe a decrease in the peak curvature position with increasing rotation up to $E k=1 \cdot 10^{-5}$, which saturates at $\kappa^{*} L=1$ for larger rotation rates (lower $E k$ ). For rotating convection a completely opposite trend is observed; a constant peak position for $E k>1.8 \cdot 10^{-4}$, where the flow is dominated by a large scale circulation (LSC) [7, 8], while the peak of curvature, $\kappa^{*}$, is slowly growing for increasing rotation rate for $E k \lesssim 1.8 \cdot 10^{-4}$, where vertically aligned vortices are the dominant flow structures [7, 8]. For $E k>1.8 \cdot 10^{-4}$, the domain-filling LSC is hardly influenced by rotation [37, which explains the constant value of $\kappa^{*}$ in the curvature PDF in this regime. For $E k \lesssim 1.8 \cdot 10^{-4}$ rotation starts to play an important role, due to the development of vertically aligned vortical plumes [7, 8 . The length scale of these vortical structures, $\mathcal{L}$, is known to decrease with increasing rotation rate [38, 39. This decrease is moreover expected to scale as $\mathcal{L} \sim E k^{1 / 3}$ [40, 41]. The trend is inversed since curvature is an inverse length: one thus expects that the curvature PDFs shift under rotation as $E k^{-1 / 3}$. In figure $4 \mathrm{~d}$ we show that for $E k<1.8 \cdot 10^{-4}$ the PDFs collapse, when the length scale is normalized by $E k^{-1 / 3}$. This collapse confirms that the PDFs shift as a whole and our chosen reference point $\kappa^{*}$ is also expected to scale as $\kappa^{*} \sim E k^{-1 / 3}$. This scaling, actually rescaled with a prefactor 


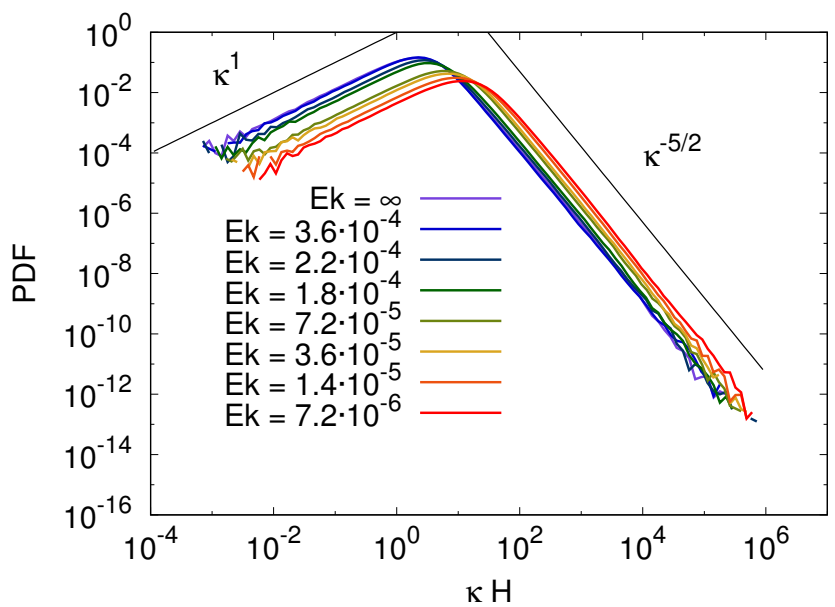

(a) experiments, top

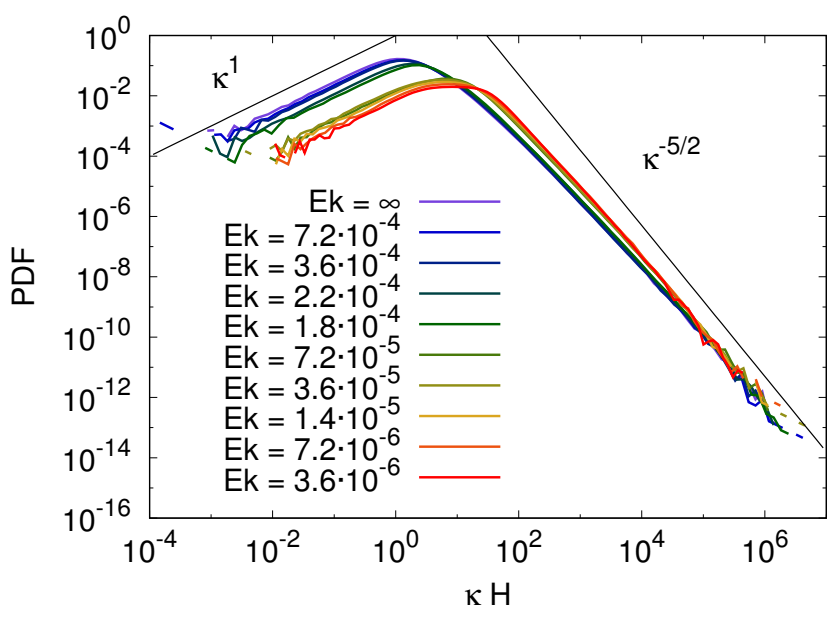

(c) numerics, only BL

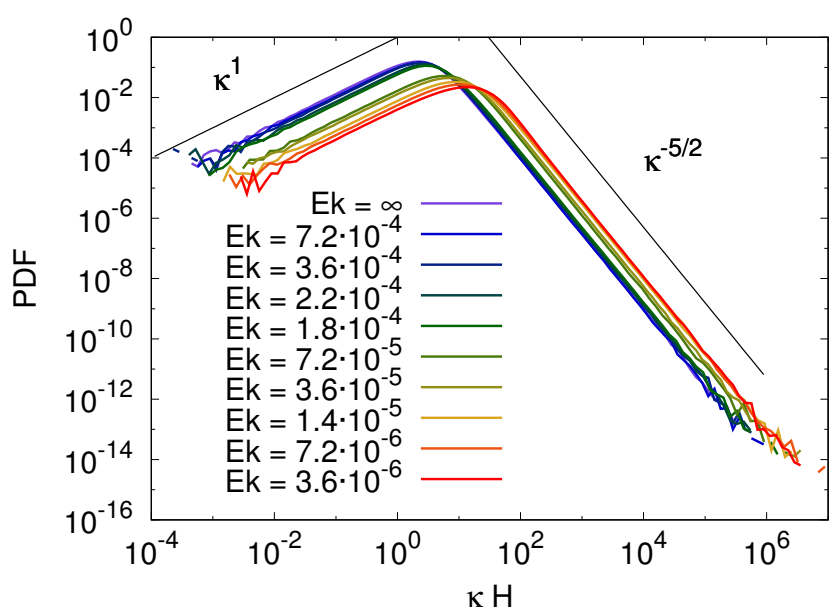

(b) numerics, top excluding BL

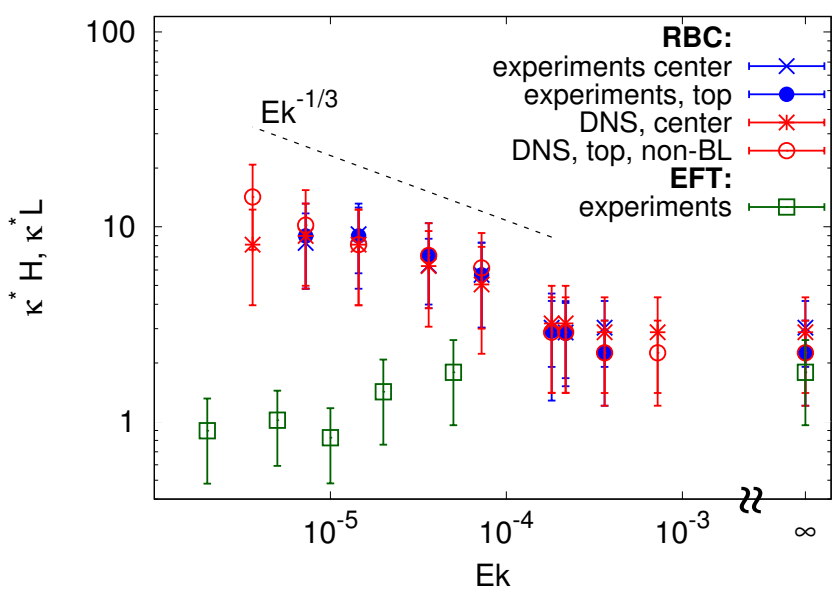

(d) $\kappa^{*}$

Figure 5: Curvature, $\kappa$, PDFs of Rayleigh-Bénard convection (RBC); (a) measured experimentally in the top measurement volume and (b)-(c) measured numerically in the non-BL region and the BL region near the to plate, respectively. Focusing on the left part of the curves, the Ekman number is increasing from bottom to top. Curvature is non-dimensionalized using the cell height, $H$. Errors in the PDFs are as discussed in figure 3 . (d) The maxima of the curvature PDFs of panel (a)-(b) and all panels of figure 4 positioned at $\kappa^{*}$, as a function of the Ekman number. For electromagnetically driven turbulence $\kappa^{*}$ is non-dimensionalized using the forcing length, $L$, and for RBC $\kappa^{*}$ is non-dimensionalized using the cell height, $H$. The error bars are given by the bin width, used to create the PDFs. The symbol 2 indicates an interruption of the $x$-axis, used to include the peak position at $E k=\infty$. Note that, consequently, this point is out of scale.

of 0.5 for the sake of figure visibility, is shown by the black dashed line in figure $5 \mathrm{~d}$ and the shift of the PDF top indeed follows this $E k^{-1 / 3}$ scaling. The effect of rotation on the typical length scale is stronger in RBC compared to EFT. In EFT the magnet array forces a length scale upon the flow, while in RBC the development of flow structures under rotation is not limited by a forcing length. When comparing the trends for RBC and EFT, we conclude that although opposite shifts for the curvature PDFs are observed in turbulent convection and electromagnetically forced turbulence, the trends are consistent with the dynamics of the vortical flow structures under rotation in the respective turbulent flows.

Also for torsion, measured numerically in turbulent convection, PDFs are computed for different Ekman numbers in all measurement volumes. In figure $6 \mathrm{a}$ and $6 \mathrm{~b}$ we again observe a shift towards higher values, both in the center and in the non-BL region near the top plate of the RBC cell. In the BL region (figure 6c we observe that also for torsion the scaling depends on the rotation rate.

The analysis of the shift of the torsion PDFs under rotation is similar as for the maximum of the curvature PDFs. However, since the torsion PDFs do not have a maximum but rather start with a constant plateau that scales as $\tau^{0}$, 


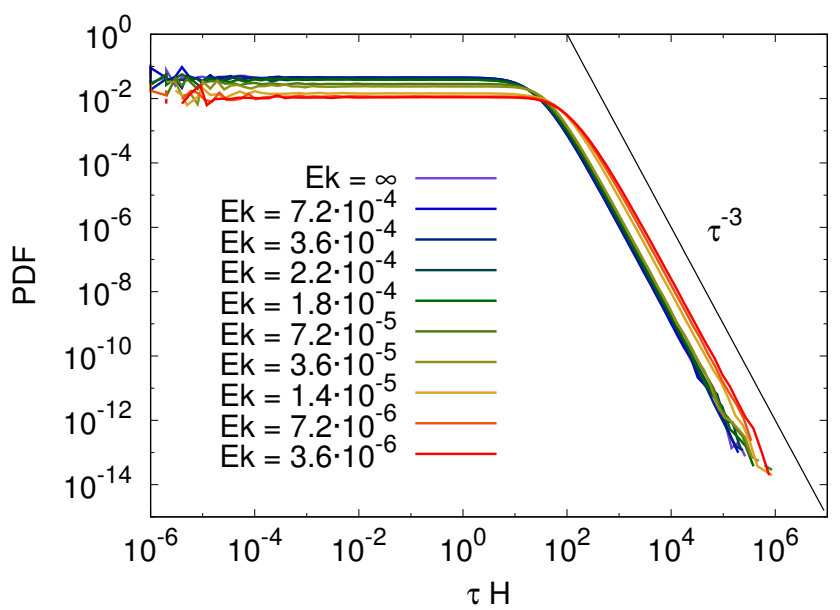

(a) numerics, center

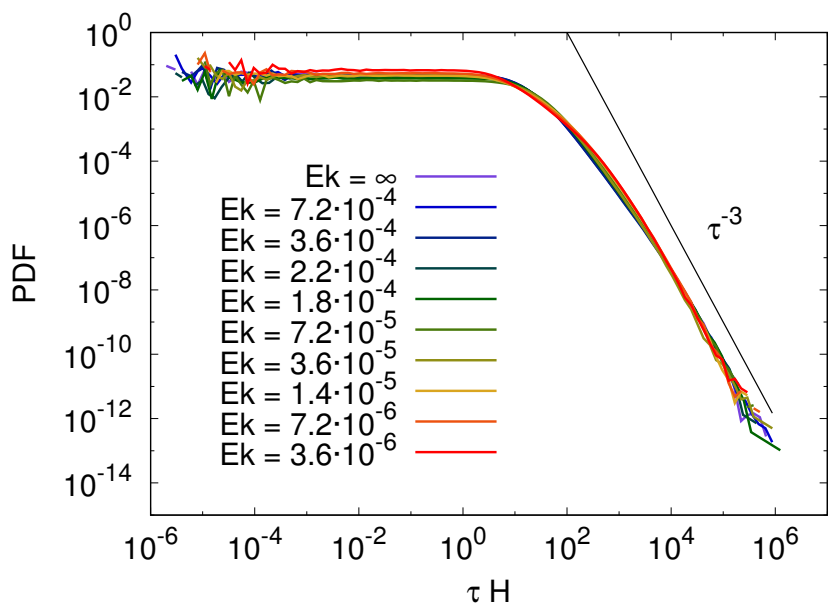

(c) numerics, only BL

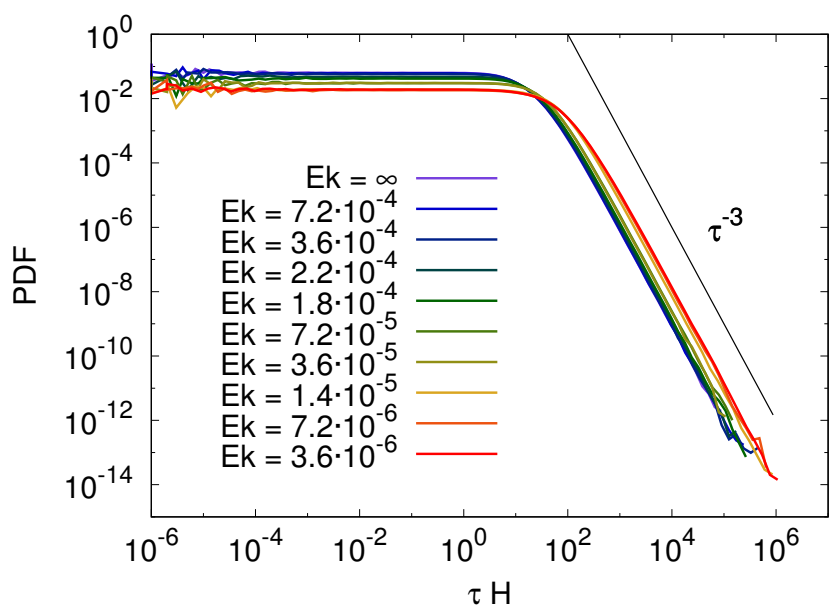

(b) numerics, top excluding BL

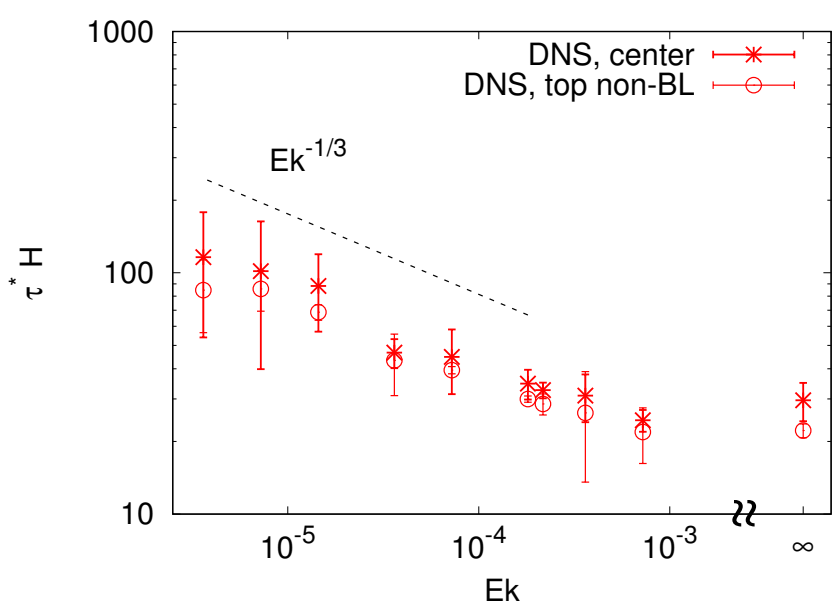

(d) $\tau^{*}$

Figure 6: (a) Torsion, $\tau$, PDFs for different Ekman numbers, measured from DNS in the center of the Rayleigh-Bénard cell. (b)-(c) Torsion PDFs of Rayleigh-Bénard convection, measured from DNS in the non-boundary layer (non-BL) and the boundary layer (BL) region near the top plate, respectively. In (a) and (b) the Ekman number is increasing from bottom to top, focusing on the left part of the curves. Errors in the PDFs are as discussed in figure 2 and 3 and torsion is non-dimensionalized using the cell height, $H$. (d) The kink position, $\tau^{*}$, corresponding to the PDFs of panels (a) and (b), as a function of the Ekman number. The value of $\tau^{*}$ is computed as the intersection point between two power law fits, performed on the constant plateau and the high torsion tail of the PDFs, respectively, and the error in $\tau^{*}$ is based on the uncertainty in these power law fits. The symbol 2 indicates an interruption of the $x$-axis, used to include $\tau^{*}$ at $E k=\infty$. Note that, consequently, this point is out of scale.

we must characterize the shift in a slightly different way. As a reference point, we now use the intersection point between the constant plateau and the $\tau^{-3}$ scaling. To compute this intersection point, $\tau^{*}$, we first perform power-law fits to the low and high torsion limits of the PDFs. Second, we compute the crossing point between these two fits. The error in $\tau^{*}$ comes from the uncertainty in the fitting results, that is dominated by the choice of the fitting range. Therefore we compute the error in the fitting exponents by shifting the fitting range first towards higher torsion values and then, towards lower torsion values, a method also used in the rest of this paper. The deviation between the fitting results in the different fitting ranges gives the error, that is then used to represent the error in $\tau^{*}$. The resulting intersection points, $\tau^{*}$, are shown as a function of the Ekman number in figure 6d. Again we first confirm that PDFs for $E k<1.8 \cdot 10^{-4}$ collapse when normalized by $E k^{-1 / 3}$, validating our expectation of similar scaling of the reference point $\tau^{*}$. We indeed observe the exact same trend as already observed for curvature: a constant PDF position in the regime of the LSC and an $E k^{-1 / 3}$ scaling in the regime where vertically aligned plumes are present.

The fact that both curvature and torsion PDFs show a horizontal shift proportional to $E k^{-1 / 3}$ (or $\Omega^{1 / 3}$ ), suggests 
that particle trajectories are 'self-similar' and that there is no stretching of trajectories in a particular direction. To understand this we use the example of the spiral, that is treated in detail in [18. The three-dimensional spiral, $\mathbf{r}(t)$, can be expressed in Cartesian coordinates as [18]:

$$
\mathbf{r}(t)=\left(\begin{array}{c}
R \cos (\Omega t) \\
R \sin (\Omega t) \\
\beta \Omega t
\end{array}\right)
$$

where $R$ and $\beta$ are the parameters describing the spiral and $\Omega$ is its angular speed. The parameters $R$ and $\beta$ can be expressed in terms of the curvature and the torsion of the spiral as [18]:

$$
R=\frac{\kappa}{\kappa^{2}+\tau^{2}}, \quad \beta=\frac{\tau}{\kappa^{2}+\tau^{2}}
$$

From equation (13) one can see that, when realizing that $\kappa$ and $\tau$ scale as $E k^{-1 / 3}$, both $R$ and $\beta$ scale as $E k^{1 / 3}$. This indeed suggests that the trajectories have a self-similar shape and do not deform under rotation. However, we realize that we are looking at statistics, rather than individual trajectories and the similar shift of PDFs for curvature and torsion is not sufficient for proving self-similarity. Random combinations of different curvature and torsion ratios are not excluded and therefore we also look at the behavior of the ratio between torsion and curvature, $\tau / \kappa$, as proposed in 21. Here it is shown that both the joint PDF of $\tau / \kappa$ with velocity and acceleration show a peak around $\tau / \kappa \approx 1$ suggesting that there is a natural ratio between torsion and curvature. In figure 7 we show these joint PDFs for non-rotating $\operatorname{RBC}(E k=\infty)$ and rotating $\operatorname{RBC}\left(E k=7.2 \cdot 10^{-6}\right)$, measured numerically in the center of the cell. Indeed we also observe that the joint PDFs have a peak around $\tau / \kappa \approx 1$, supporting the hypothesis of self-similarity of trajectories.

Next, we focus on the PDFs measured both in the boundary layers and in the bulk, and analyze the effect of rotation on their scaling behavior. We focus on the high-curvature and high-torsion tails of the PDFs, where the scaling is indicated by $\kappa^{-c_{1}}$ and $\tau^{-c_{2}}$ for curvature and torsion, respectively. To identify a trend in the scaling exponents $c_{1}$ and $c_{2}$, we measure them directly from a power-law fit. The results are shown as a function of the Ekman number in figure 8 for all measurement volumes, where the errors in the exponents are determined by comparing different fitting ranges as discussed above. It is clearly observed that in the cell center the scaling is unaffected by rotation for both curvature and torsion and equals the HIT scaling, corresponding to $c_{1}=5 / 2$ and $c_{2}=3$. Also, in the non-BL region near the top plate, a constant scaling is observed equal to this HIT prediction. However, in the boundary layer the scaling exponent decreases for decreasing rotation rate or increasing $E k$, for both curvature and torsion. Remarkable is that for higher rotation rates the HIT scaling is approached, while the lower the rotation rate, the more the exponents deviate from this prediction. It is known that at $E k=1.8 \cdot 10^{-4}$, the transition point between LSC and vertically aligned vortices, there is also a transition in the boundary layer dynamics: for $E k>1.8 \cdot 10^{-4}$, a Prandtl-Blasius boundary layer develops at the horizontal plates, while for $E k \lesssim 1.8 \cdot 10^{-4}$ the boundary layer is of the Ekman type [13. For $E k>1.8 \cdot 10^{-4}$, the scaling exponents of the PDFs in the BL are constant and reach the minimum values $c_{1} \approx 2.2 \pm 0.1$ for curvature and $c_{2} \approx 2.5 \pm 0.2$ for torsion. The Prandtl-Blasius BL is not influenced by rotation 13 , which explains the constant scaling exponent in this regime. Moreover the Prandtl-Blasius BL is passive and is hardly influenced by the bulk, consistent with the deviation of the scaling exponent from the bulk value in this regime. To understand why the scaling exponents in the Prandtl-Blasius BL are lower than the HIT prediction, we go back to the derivations of the scaling laws in section II. Here, we focused on HIT and assumed that the velocity components were independent Gaussian variables, following a $\chi$-distribution of dimension 3. Although the velocity statistics in $\mathrm{RBC}$ are not perfectly Gaussian, especially near the top plate and in the BL, this assumption is reasonable for small values of $u$, which is exactly the limit we are interested in (see also Section IV A). Using this assumption, $u^{-2}$ follows an inverse $\chi$-squared distribution of dimension $k=3$. However, in the Prandtl-Blasius boundary layer, there is just one dominant horizontal velocity component due to the presence of the mean flow [27]. As a result the number of velocity components can be thought of as being effectively reduced from 3 to $3-\gamma$, where $\gamma$ indicates a fractional reduction of the dimensionality of the flow. Expanding the distribution of $u^{-2}$ (equation (3)) with this new dimension $k=3-\gamma$, to $x=u^{-2}$ for $x \rightarrow \infty$, results in $P_{u^{-2}}\left(x=u^{-2}\right) \sim x^{-(5 / 2-\gamma / 2)}$. This means that in the limit of $\kappa \rightarrow \infty$ the curvature PDF scales with scaling exponent $c_{1}=5 / 2-\gamma / 2$. While for $\gamma=0$ the HIT value of $c_{1}=5 / 2$ is recovered, the exponent decreases with increasing $\gamma$. For torsion we rewrite the integral of equation (5), now assuming $a_{n}$ and $u$ to follow a $\chi$-distribution of dimension $2-\gamma$ and $3-\gamma$, respectively [19]:

$$
P_{\tau}(\tau) \sim \iint u^{2-\gamma} \mathrm{e}^{-\frac{u^{2}}{2}} a_{n}^{1-\gamma} \mathrm{e}^{-\frac{a_{n}^{2}}{2}} \delta\left(\tau-\frac{1}{a_{n} u}\right) d a_{n} d u
$$

Following the same steps as in section II (equations (5) - (8)), results in a scaling of $P_{\tau}(\tau) \sim \tau^{-(3-\gamma)}$, for $\tau \rightarrow \infty$, thus $c_{2}=3-\gamma$. Indeed, a decrease in the number of velocity components from 3 to $3-\gamma$, leads to lower scaling 


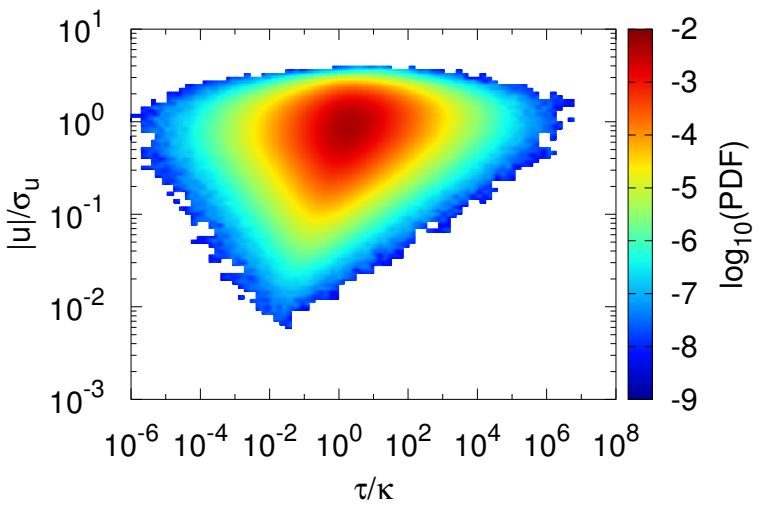

(a) $E k=\infty$

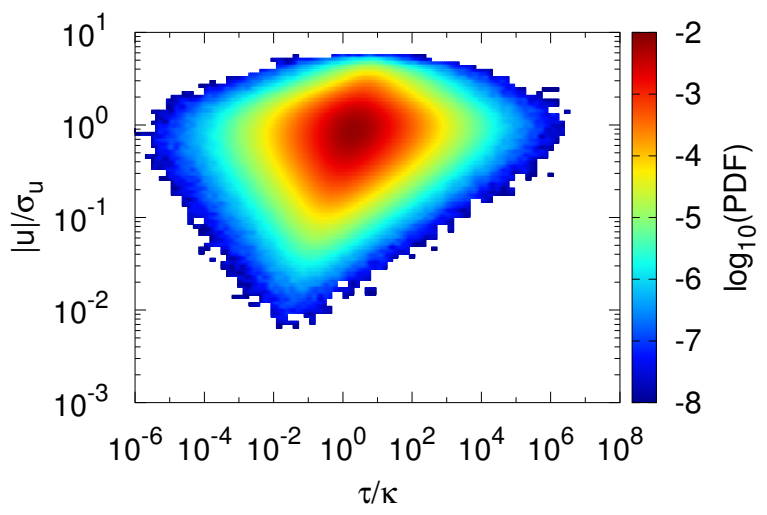

(c) $E k=7.2 \cdot 10^{-6}$

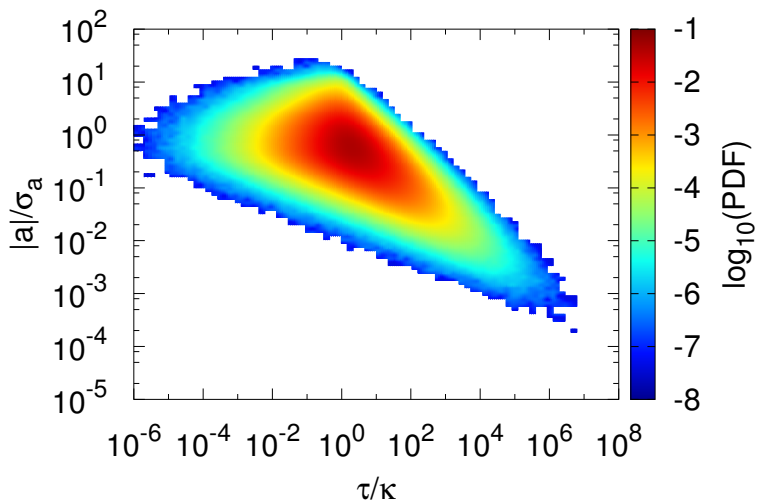

(b) $E k=\infty$

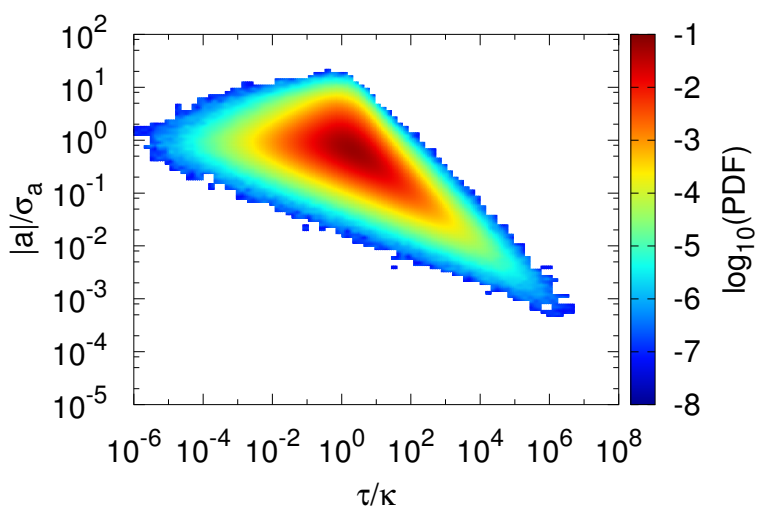

(d) $E k=7.2 \cdot 10^{-6}$

Figure 7: Joint PDFs of the ratio between torsion and curvature, $\tau / \kappa$, with (a) absolute velocity $|u|$ and (b) absolute acceleration $|a|$, measured numerically in the center of the Rayleigh-Bénard cell for $E k=\infty$. The velocity and acceleration are normalized by their standard deviations, $\sigma_{u}$ and $\sigma_{a}$ respectively. In panel (c) and (d) these joint PDFs are shown for $E k=7.2 \cdot 10^{-6}$

exponents for both curvature and torsion PDFs. As $E k \rightarrow \infty$ the scaling exponents $c_{1}$ and $c_{2}$ converge to constant values $c_{1}=2.2 \pm 0.1$ and $c_{2}=2.5 \pm 0.2$, corresponding to $\gamma=0.5 \pm 0.1$. Although this value of $\gamma$ is found in both the curvature and torsion measurements, we are not aware of any theoretical explanation or previous studies quantifying this reduction in dimensionality.

Different from the Prandtl-Blasius boundary layer, the Ekman boundary layer does actively interact with the bulk flow. Moreover, the vertical velocity coupling bulk and boundary layer (also known as Ekman pumping/suction), scales with the Ekman number [42, 43, and consequently curvature depends on the rotation rate as well. Indeed we observe that the scaling exponents depend on rotation in figure 8 and are gradually increasing towards the bulk values of $c_{1}=5 / 2$ and $c_{2}=3$ for higher rotation rates, as expected because of the active interplay between the Ekman boundary layer and the bulk (where HIT scaling is observed).

\section{CONCLUSIONS}

We studied the effect of rotation on the geometry of tracer trajectories in two different types of rotating turbulent flows: Rayleigh-Bénard convection and (isothermal) turbulence driven by electromagnetic forcing. We found that 


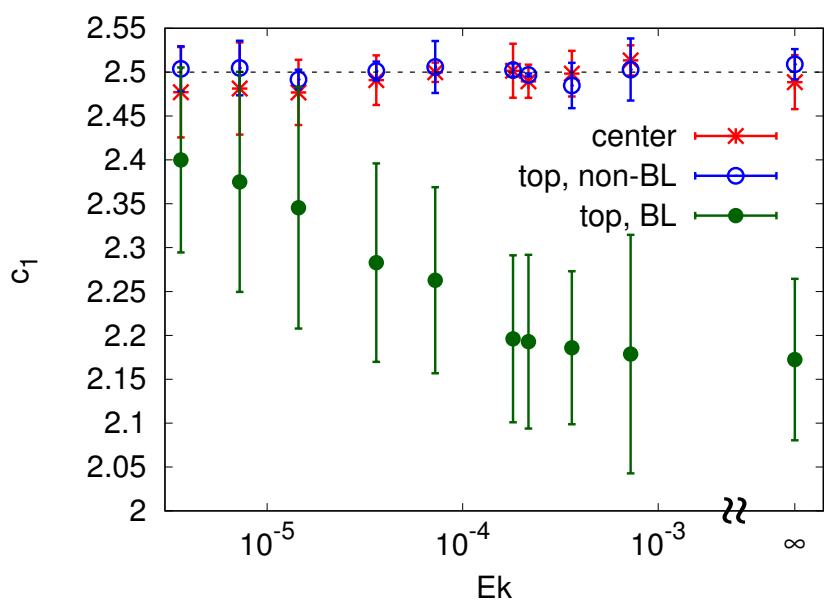

(a) curvature

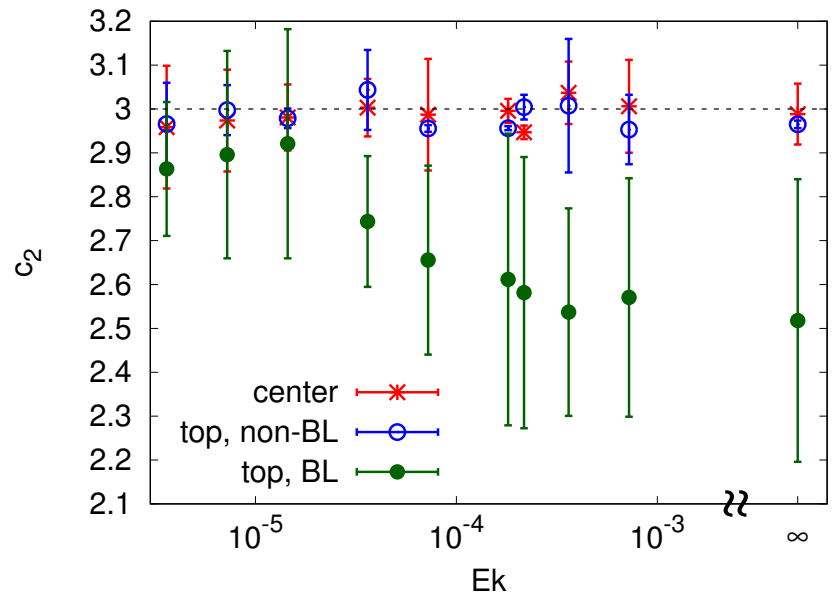

(b) torsion

Figure 8: (a) Scaling exponents $c_{1}$, corresponding to the high curvature scaling $\kappa^{-c_{1}}$, and (b) $c_{2}$, corresponding to the high torsion scaling $\tau^{-c_{2}}$, as a function of the Ekman number, measured numerically in both the center, the non-boundary layer (non-BL) and boundary layer (BL) regions near the top plate of the convection cell. Error bars are determined by comparing different fitting ranges: one shifted towards higher curvature and torsion values and another one shifted towards lower values of the concerned PDF tail. The symbol 2 indicates an interruption of the $x$-axis, used to include the scaling exponents at $E k=\infty$. Note that, consequently, this point is out of scale.

the response of curvature and torsion statistics to rotation is connected to the length scales of typical flow structures, regardless of the type of turbulence, hence showing some degree of universality. In electromagnetically driven turbulence, the length scales of vortical structures increase with increasing rotation rate and lower curvature is expected for higher rotation rates. This shift towards lower curvature values is indeed observed in the curvature PDFs measured in the EFT experiments. In RBC the typical flow structure is a large-scale circulation for rotation rates below a critical value, $E k=1.8 \cdot 10^{-4}$. Here, rotation does not play a significant role and indeed the curvature statistics are found to be unaffected by rotation in this regime. For rotation rates above this critical value, or correspondingly $E k \lesssim 1.8 \cdot 10^{-4}$, vertically aligned vortical plumes develop and their length scale decreases with increasing rotation rate, exactly opposite to the trend observed in electromagnetically forced turbulence. In RBC curvature and torsion PDFs indeed shift towards higher values, both in the center of the cell and near the top plate. As a result the effect of rotation on the geometry of particle trajectories is consistent for the two completely different turbulence forcing methods.

The scaling of the tails of the PDFs perfectly matches the scaling laws previously found in homogeneous isotropic turbulence (HIT) for all rotation rates and both types of turbulent flows, except for the boundary layers. This is somewhat surprising, since in both EFT and RBC the vertical velocity statistics are certainly not perfectly Gaussian and moreover the bulk flow is anisotropic and inhomogeneous. We realize, however, that statistics can again be assumed Gaussian in the limit of very small velocity and that anisotropy is strongest inside the boundary layer. So, as long as the flow is turbulent and viscosity does not play a role, the scaling laws of curvature and torsion PDFs are independent of the forcing mechanism, very robust and not influenced by anisotropy.

In the DNS of RBC, we were able to study the dynamics of trajectories inside the viscous boundary layer, where the flow dynamics is completely different from the turbulent bulk. Our results reveal that, in the viscous boundary layer, a lower scaling exponent is found for lower rotation rates, while the HIT prediction is approached for higher rotation rates. For $E k>1.8 \cdot 10^{-4}$, a Prandtl-Blasius boundary layer is present at the horizontal plates and the number of independent velocity components is effectively decreased due to the presence of a mean horizontal flow. Taking this into account, while expanding the probability distributions of curvature and torsion in the limit of high curvature and torsion, indeed explains the lower scaling exponents in this regime. For $E k \lesssim 1.8 \cdot 10^{-4}$, the boundary layer is of the Ekman type; its dynamics is actively driven by the bulk flow (different from the passive Prandtl-Blasius boundary layer) and the velocity depends on the Ekman number. Indeed also the scaling exponents depend on the Ekman number and they gradually increase from the exponents observed in the regime of the Prandtl-Blasius boundary layer, towards the HIT prediction, with increasing rotation rate. This indicates that, in the case of active coupling between bulk flow and the Ekman boundary layer, rotation suppresses anisotropy.

So, as long as viscosity does not play a role and the flow is turbulent, the scaling laws for curvature and torsion PDFs 
are independent of rotation rate and forcing method and moreover not influenced by anisotropy or inhomogeneity. However, as soon as we step into the viscous boundary layer and the statistical properties of turbulence do not hold anymore, the scaling exponents react on rotation, consistent with the type of boundary layer in the different rotational regimes.

\section{ACKNOWLEDGMENTS}

This work is supported by the Stichting voor Fundamenteel Onderzoek der Materie (FOM), the Netherlands. The authors gratefully acknowledge the support of the Nederlandse Organisatie voor Wetenschappelijk Onderzoek (NWO) for the use of supercomputer facilities (Cartesius) under Grant No. SH-334-15. EU-COST action MP1305 'Flowing matter' is kindly acknowledged.

[1] J. Marshall and F. Schott, "Open-ocean convection: Observations, theory, and models," Rev. Geophys. 37, 1-64 (1999).

[2] M. S. Miesch, "The Coupling of solar convection and rotation," Solar Phys. 192, 59-89 (2000).

[3] M. G. Dunn, "Convective heat transfer and aerodynamics in axial flow turbines," J. Turbomach 123, 637-686 (2001).

[4] J. P. Johnston, "Effect of system rotation on turbulence structure: a review relevant to turbomachinery flows," Int. J. Rotating Mach. 4, 97-112 (1998).

[5] L. J. A. van Bokhoven, H. J. H. Clercx, G. J. F. van Heijst, and R. R. Trieling, "Experiments on rapidly rotating turbulent flows," Phys. Fluids 21, 096601 (2009).

[6] E. J. Hopfinger, F. K. Browand, and Y. Gagne, "Turbulence and waves in a rotating tank," J. Fluid Mech. 125, 505-534 (1982).

[7] R. J. A. M. Stevens, H. J. H. Clercx, and D. Lohse, "Heat transport and flow structure in rotating Rayleigh-Bénard convection," Eur. J. Mech. B/Fluids 40, 41-49 (2013).

[8] R. P. J. Kunnen, H. J. H. Clercx, and B. J. Geurts, "Breakdown of large-scale circulation in turbulent rotating convection," Europhys. Lett. 84, 24001 (2008).

[9] Y. Liu and R. E. Ecke, "Heat transport scaling in turbulent Rayleigh-Bénard convection: Effects of rotation and Prandtl number," Phys. Rev. Lett. 79, 2257-2260 (1997).

[10] H. T. Rossby, "A study of Bénard convection with and without rotation," J. Fluid Mech. 36, 309-335 (1969).

[11] R. J. A. M. Stevens, H. J. H. Clercx, and D. Lohse, "Optimal Prandtl number for heat transfer in rotating Rayleigh-Bénard convection," New J. Phys. 12, 075005 (2010).

[12] J.-Q. Zhong, R. J. A. M. Stevens, H. J. H. Clercx, R. Verzicco, D. Lohse, and Ahlers G., "Prandtl-, Rayleigh-, and Rossby-number dependence of heat transport in turbulent rotating Rayleigh-Bénard convection," Phys. Rev. Lett. 102, 044502 (2009).

[13] H. Rajaei, P. Joshi, K. M. J. Alards, R. P. J. Kunnen, F. Toschi, and H. J. H. Clercx, "Transitions in turbulent rotating convection: A Lagrangian perspective," Phys. Rev. E 93, 043129 (2016).

[14] R. J. A. M. Stevens, J.-Q. Zhong, H. J. H. Clercx, G. Ahlers, and D. Lohse, "Transitions between turbulent states in rotating Rayleigh-Bénard convection," Phys. Rev. Lett. 103, 024503 (2009).

[15] P. Wei, S. Weiss, and G. Ahlers, "Multiple transitions in rotating turbulent Rayleigh-Bénard convection," Phys. Rev. Lett. 114, 114506 (2015).

[16] M. A. Green, C. W. Rowley, and G. Haller, "Detection of Lagrangian coherent structures in three-dimensional turbulence," J. Fluid Mech. 572, 111-120 (2007).

[17] J. Schumacher, "Lagrangian dispersion and heat transport in convective turbulence," Phys. Rev. Lett. 100, 134502 (2008).

[18] W. Braun, F. De Lillo, and B. Eckhardt, "Geometry of particle paths in turbulent flows," J. Turbul. 7, N62 (2006).

[19] A. Scagliarini, "Geometric properties of particle trajectories in turbulent flows," J. Turbul. 12, N25 (2011).

[20] H. Xu, N. T. Ouellette, and E. Bodenschatz, "Curvature of Lagrangian trajectories in turbulence," Phys. Rev. Lett. 98, $050201(2007)$.

[21] Y. Choi, Y. Park, and C. Lee, "Helicity and geometric nature of particle trajectories in homogeneous isotropic turbulence," Int. J. Heat Fluid Fl. 31, 482-487 (2010).

[22] L. Del Castello and H. J. H. Clercx, "Lagrangian velocity autocorrelations in statistically steady rotating turbulence," Phys. Rev. E 83, 056316 (2011).

[23] R. P. J. Kunnen, B. J. Geurts, and H. J. H. Clercx, "Experimental and numerical investigation of turbulent convection in a rotating cylinder," J. Fluid Mech. 642, 445-476 (2010).

[24] R. P. J. Kunnen, H. J. H. Clercx, and B. J. Geurts, "Enhanced vertical inhomogeneity in turbulent rotating convection," Phys. Rev. Lett. 101, 174501 (2008).

[25] H. Rajaei, P. Joshi, R. P. J. Kunnen, and H. J. H. Clercx, "Flow anisotropy in rotating buoyancy-driven turbulence," Phys. Rev. Fluids 1, 044403 (2016).

[26] C. Sun, Y.-H. Cheung, and K.-Q. Xia, "Experimental studies of the viscous boundary layer properties in turbulent Rayleigh-Bénard convection," J. Fluid Mech. 605, 79-113 (2008). 
[27] O. Shishkina, S. Horn, and S. Wagner, "Falkner-Skan boundary layer approximation in Rayleigh-Bénard convection," J. Fluid Mech. 730, 442-463 (2013).

[28] P. M. Lee, Bayesian statistics: An introduction (Arnold, New York, 2004).

[29] B. Lüthi, A. Tsinober, and W. Kinzelbach, "Lagrangian measurement of vorticity dynamics in turbulent flow," J. Fluid Mech. 528, 87-118 (2005).

[30] J Willneff, "3D particle tracking velocimetry based on image and object space deformation," Int. Arch. Photogramm. Rem. Sens. Spatial Inform. Sci. 34, 601-606 (2002).

[31] B. Lüthi, Some aspects of strain, vorticity and material element dynamics as measured with 3D particle tracking velocimetry in a turbulent flow, Ph.D. thesis, Swiss Federal Institue of Technology, Switzerland (2002).

[32] R. Verzicco and R. Camussi, "Numerical experiments on strongly turbulent thermal convection in a slender cylindrical cell," J. Fluid Mech. 477, 19-49 (2003).

[33] R. Verzicco and Orlandi P., "A finite-difference scheme for three-dimensional incompressible flows in cylindrical coordinates," J. Comput. Phys. 123, 402-414 (1996).

[34] L. Del Castello and H. J. H. Clercx, "Lagrangian acceleration of passive tracers in statistically steady rotating turbulence," Phys. Rev. Lett. 107, 214502 (2011).

[35] Q. Zhou, C. Sun, and K.-Q. Xia, "Experimental investigation of homogeneity, isotropy, and circulation of the velocity field in buoyancy-driven turbulence," J. Fluid Mech. 598, 361-372 (2008).

[36] L. Del Castello, Table-top rotating turbulence : an experimental insight through Particle Tracking, Ph.D. thesis, Eindhoven University of Technology, Eindhoven (2010).

[37] J.-Q. Zhong and G. Ahlers, "Heat transport and the large-scale circulation in rotating turbulent Rayleigh-Bénard convection," J. Fluid Mech. 665, 300-333 (2010).

[38] P. Vorobieff and R. E. Ecke, "Vortex structure in rotating Rayleigh-Bénard convection," Physica D 123, 153-160 (1998).

[39] R. P. J. Kunnen, H. J. H. Clercx, and B. J. Geurts, "Vortex statistics in turbulent rotating convection," Phys. Rev. E 82, 036306 (2010).

[40] S. Chandrasekhar, Hydrodynamic and Hydromagnetic Stability (Dover Publications, New York, 1961).

[41] E. M. King, S. Stellmach, and B. Buffett, "Scaling behaviour in Rayleigh-Bénard convection with and without rotation," J. Fluid Mech. 717, 449-471 (2013).

[42] V. W. Ekman, "On the influence of the Earth's rotation on ocean currents," Ark. Mat. Astron. Fys. 2, 1-53 (1905).

[43] H. P. Greenspan, The theory of rotating fluids (Cambridge University Press, Cambridge, 1968). 